\title{
Lösungsmöglichkeiten bei fehlgeschlagenen Osteosynthesen in der Mund-, Kiefer- und Gesichtschirurgie
}

Steffen Koerdt, Antonis Sabatakakis, Max Heiland, Kilian Kreutzer

\author{
Die Plattenosteosynthese hat sich in der Mund-, Kiefer- und Gesichtschirurgie für die \\ Frakturversorgung als Standard etabliert. Die anatomisch-ästhetischen Einschrän- \\ kungen der extraoralen und intraoralen Zugangswege im Gesichtsbereich haben \\ einen Einfluss auf die Frakturversorgung. Die unmittelbare Belastung durch Kau- \\ kräfte, die anatomische Lagebeziehung zur Mundhöhle und zum Nasennebenhöh- \\ lensystem und die Einbeziehung der Okklusion sind Faktoren, die zu einem Miss- \\ erfolg der Osteosynthese führen können.
}

\section{Die Frakturbehandlung in der Mund-, Kiefer- und Gesichtschirurgie}

Die Traumatologie stellt eine zentrale Säule innerhalb des klinischen Behandlungsspektrums im Fachgebiet „Mund-, Kiefer- und Gesichtschirurgie“ dar. Knöcherne Verletzungen des Gesichtsschädels zählen zu den häufigsten Frakturen des Menschen [1]. Knöcherne Verletzungen des Mittelgesichtes lassen sich in zentrale, laterale und zentrolaterale Frakturen unterteilen. Frakturen des Unterkiefers, meist an den Prädilektionsstellen im Bereich der Eckzahnwurzel, am Foramen mentale, am Kieferwinkel oder am Gelenkfortsatz, stellen die zweithäufigste Art von Gesichtsschädelfrakturen dar. Darüber hinaus sind Frakturmuster wie die isolierten Nasenbeinfrakturen, die Frakturen des Alveolarfortsatzes und ausgedehnte panfaziale Traumata mit unterschiedlichen Kombinationsmöglichkeiten der einzelnen Frakturen zu erwähnen. Sonderfälle im Fachbereich der Mund-, Kiefer- und Gesichtschirurgie sind pathologische Frakturen infolge von Grunderkrankungen wie einem ossär metastasierten Tumorleiden, Osteoporose oder aufgrund einer Alveolarkammatrophie sowie Frakturen im Kindesalter.

Der reproduzierbare Vielpunktkontakt, also die maximale Interkuspidation zwischen Ober- und Unterkiefer, in der Zahnmedizin als Okklusion bezeichnet, bestimmt in der Therapie von Frakturen des Gesichtsschädels das Repositionsergebnis. Die Einstellung der Okklusion erfolgt durch eine mandibulomaxilläre Fixation (MMF), bei der die Zähne des Ober- und Unterkiefers entweder mit lockeren oder straffen Gummizügen oder Drahtligaturen aufeinandergepresst werden. Hierzu bedarf es jedoch aufgrund der speziellen Anatomie im Kieferbereich der Eingliederung von Drahtschienen mit Einzelzahndrahtligaturen,
Fixationsschrauben oder zahntechnisch hergestellten Geräten. Durch eine MMF, sei es im Rahmen der konservativen Therapie für einen gewissen Zeitraum oder nur temporär im Rahmen der operativen Versorgung, kann über eine regelhafte Okklusion die anatomisch korrekte Reposition der Frakturenden erreicht werden. Die konservative Frakturbehandlung, auch wenn seit Jahrhunderten so durchgeführt [2] folgt in Analogie zur offenen, chirurgischen Frakturbehandlung den Prinzipien der Reposition und Retention [3] und ist heutzutage weitestgehend in den Hintergrund getreten und nur speziellen Indikationen vorbehalten [4].

Die operative Frakturbehandlung erfolgt auch in der Mund-, Kiefer- und Gesichtschirurgie dem Konzept des „open reduction and internal fixation" (ORIF) [5]). Dabei werden die Frakturlinien über einen operativen Zugang dargestellt, die offene Reposition durchgeführt und das Ergebnis mittels Plattenosteosynthese gesichert. Hierbei wird vor allem Titan und entsprechende Schrauben aus einer härteren Titan-Aluminium-Vanadium-Legierung verwendet [6-8].

Der operative Zugangsweg zum Gesichtsschädel richtet sich nach der Lokalisation der Fraktur bzw. des Defektes und kann von transoral, transfazial, transkonjunktival oder über die den einzelnen Indikationen entsprechenden Zugangswege wie den bikoronaren Bügelschnitt ( $>$ Abb. 1) bzw. den prä- oder retroaurikulären Zugang reichen. Auch eine Kombination mehrerer Zugangswege ist oft sinnvoll und notwendig, um eine Schädigung des N. facialis oder des N. trigeminus zu verhindern.

Bei der Frakturversorgung kann unterschieden werden zwischen einer übungsstabilen Osteosynthese, die eine sofortige passive Bewegung erlaubt, und einer funktions- 


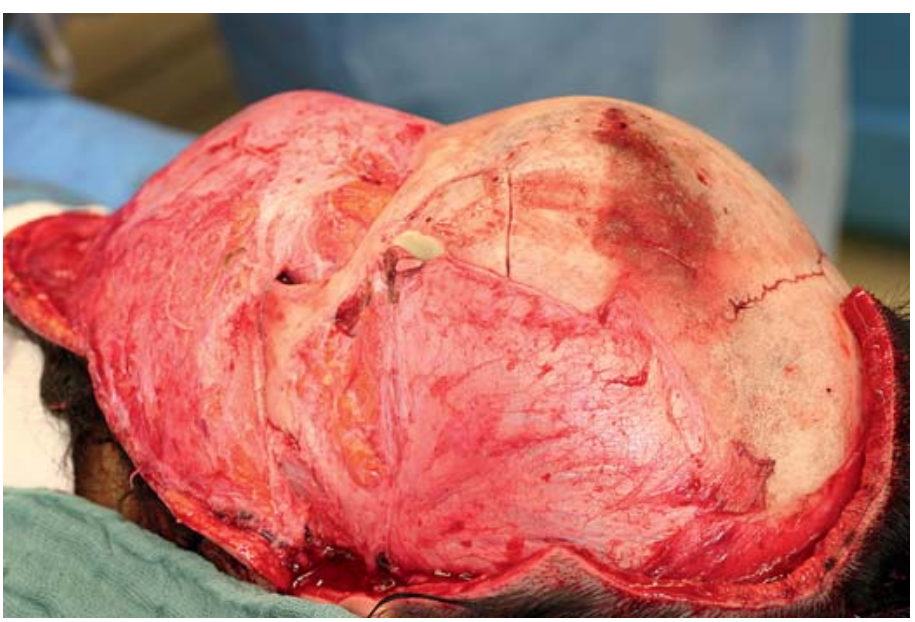

- Abb. 1 Klinisches Bild nach Bügelschnitt zur Rekonstruktion des Mittelgesichtes.

stabilen Osteosynthese, die eine sofortige Kaubelastung ermöglicht. Von entscheidender Bedeutung ist zudem, ob die Kräfte über den Frakturspalt nur durch das Osteosynthesematerial (load-bearing) oder zusätzlich auch über eine knöcherne Abstützung (load-sharing) übertragen werden.

Die Osteosynthese im nicht lasttragenden Bereich der Stirn, der Orbita und des Jochbogens erfolgt i.d.R. mittels einer funktionsstabilen Miniplattenosteosynthese oder in Ausnahmefällen mit resorbierbarem Osteosynthesematerial [9]. Im lasttragenden Bereich des Oberkiefers und Jochbeins kommt meistens eine Miniplattenosteosynthese entlang der Trajektorien, also der knöchernen Spannungslinien, die die Kaukräfte in Richtung der Schädelbasis übertragen, zum Einsatz.

Die regelhafte Versorgung von Unterkieferfrakturen stellt die größte Herausforderung dar. Hier sind mehrere Faktoren in die Auswahl der richtigen Osteosynthese einzubeziehen. Die Bandbreite der Versorgung reicht über eine Miniplattenversorgung mit einer oder mehreren Platten entlang der Zug- und Druckspannungslinien [10] bis hin zum Einbringen einer Rekonstruktionsplatte bei Trümmerfrakturen über einen ausgedehnten extraoralen Zugang. Auch die Fixierung autologer ossärer Transplantate zur Rekonstruktion nach ablativer Tumorchirurgie, ausgedehnten Entzündungen, Osteoradionekrose oder Traumata erfolgt mithilfe von Osteosynthesematerial.

\section{Komplikationen bei Osteosynthesen}

Wie in der allgemeinen Traumatologie können osteosynthetische Verfahren auch in der Mund-, Kiefer- und Gesichtschirurgie mit unterschiedlichen Herausforderungen und Komplikationen einhergehen. Insgesamt besteht eine geringe Komplikationsrate von ca. 10\% [11,12]. Zu den häufigen Komplikationen gehören: Materiallockerung, Materialbruch, sekundäre Fragmentdislokation und Infektion. Die Ursachen sind dementsprechend in der Auswahl der knöchernen Verankerungspunkte, der Auswahl des Osteosynthesematerials, der biomechanischen Belastung des Osteosynthesematerials und der Weichteildeckung zu sehen.

Infektionen von eingebrachtem Osteosynthesematerial sind eine häufige Komplikation. Über die häufig verwendeten intraoralen Zugänge ist eine bakterielle Kontamination des Osteosynthesematerials unvermeidbar. Über eine in den Parodontalspalt mündende Frakturlinie steht das Osteosynthesematerial bei Frakturen, die die Zahnreihe betreffen, auch langfristig in Kontakt zur Mundhöhle. Gerade bei ausgedehnten Traumata mit ausgedehnten Lazerationen der Mundschleimhaut ist ein bakteriendichter Wundverschluss zur Mundhöhle oft nicht zu realisieren. Auch eine Keimbesiedelung im Bereich der Kieferhöhlen und der Orbitae im Rahmen des Traumas kann zur Folge haben, dass das Osteosynthesematerial in einen kontaminierten Bereich eingebracht werden muss. Es ist jedoch zu beachten, dass sich in den seltensten Fällen eine klinische Konsequenz ergibt, da die gute Durchblutung und immunologische Abwehrlage im Bereich der Mundhöhle zu einer reizlosen Wundheilung führen.

Einen wichtigen Faktor stellt die Verankerung der Osteosyntheseplatten durch Schrauben dar. Sowohl eine ausreichende Anzahl an Schrauben zur Verteilung der Kräfte als auch die Wahl des Knochenlagers sind entscheidend für eine erfolgreiche Osseointegration.

Probleme im Hinblick auf die Frakturheilung können bei Patienten mit Störungen des Knochenstoffwechsels auftreten. Speziell Kiefernekrosen nach antiresorptiver Therapie aufgrund von metastasierten Tumorerkrankungen oder hämatologischen Grunderkrankungen stellen Herausforderungen in der chirurgischen Therapie dar. In diesem Zusammenhang seien außerdem Strahlenfolgen im Sinne einer Osteoradionekrose und strahleninduzierten Fibrose der Haut und Schleimhäute zu nennen, die eine osteosynthetische Versorgung bei diesem Patientenkollektiv erschweren.

Eine besondere Herausforderung sind Trümmerfrakturen der lasttragenden Kieferabschnitte. Sowohl im Ober- als auch im Unterkiefer können hierbei jegliche Möglichkeiten der Verankerung aufgehoben sein. Während man im Oberkiefer hier eine Teilkonsolidierung abwarten kann, ist im Unterkiefer die Indikation zur Implantation eines Fixateurs externe ( $\bullet$ Abb. 2 ) gegeben, um eine Ruhigstellung der verbliebenen Knochenfragmente zu erreichen.

Im umgekehrten Fall tritt ein Bruch von Osteosyntheseplatten eigentlich nur bei nicht ausreichend dimensionierten Platten auf [13]. Dies ist meistens in der primären 
Auswahl des Osteosynthesematerials begründet. Allerdings können auch Parafunktionen (Knirschen, Pressen), Noncompliance in Bezug auf die Nahrungsaufnahme und eine insuffiziente Reposition zu höheren Belastungen führen. Eine operative Revision mit Erweiterung des operativen Zugangs und Einbringen einer kräftigeren Osteosyntheseplatte ist unumgänglich.

Kommt es dabei zu keinem Bruch des Osteosynthesematerials, stellt sich lediglich eine erhöhte Mobilität mit Fragmentdislokation ein. Oft ist dabei eine falsche Lage der Platte in Bezug auf die die Kaukräfte aufnehmenden Pfeiler oder die Verwendung von zu wenig Osteosyntheseplatten ursächlich. So kann bspw. die Rotationsstabilität bei Unterkieferfrakturen falsch eingeschätzt werden. Der Verzicht auf eine 2. Platte führt dann zum Abgleiten der Frakturenden. Als Folge kommt es zur Ausbildung einer Pseudoarthrose mit persistierender Beweglichkeit über den Frakturspalt und der Gefahr der Ausbildung einer lokalen Infektion oder gar einer Osteomyelitis.

\section{Kasuistiken}

Im Weiteren möchten wir anhand von 4 unterschiedlichen Patientenfällen das Komplikationsmanagement bei Osteosynthesen im Bereich der Mund-, Kiefer- und Gesichtschirurgie näher vorstellen.

\section{Fall 1}

Der 55-jährige Patient stellt sich nach Unterkieferkontinuitätsresektion, modifiziert radikaler Neck Dissection und Rekonstruktion mittels mikrovaskulär reanastomosiertem Fibulatransplantat und interner Fixation mittels 6 Miniplatten bei Z.n. adjuvanter Strahlentherapie und Plattenbruch von 4 der eingebrachten 6 Miniplatten in der Klinik vor ( $\vee$ Abb. $\mathbf{3}$ a, b). Bei klinisch eingeschränkter Mundöffnung und einem ausgeprägten Fehlbiss entschlossen wir uns zur Revision der Pseudoarthrosen, Verkürzung des aufsteigenden Unterkieferastes und Fixation des zuvor eingebrachten Fibulatransplantates mittels eines patientenspezifischen Implantates (PSI) im Sinne einer individuell gesinterten im CAD-/CAM-Verfahren hergestellten 2,7-mm-Titanplatte ( $\bullet$ Abb. $3 \mathbf{c}$ ).

\section{Fall 2}

In 12/2016 wurde bei dem heute 56-jährigen Patienten eine 2-fache Unterkieferfraktur im Bereich der Gelenkfortsatzbasis rechts und im Bereich des Unterkieferkorpus links osteosynthetisch mit 2 4-Loch-Miniplatten am Collum und einer 6-Loch-Miniplatte am Unterkieferkorpus versorgt ( Abb. 4 a). Innerhalb von 4 Wochen wurde bei Bruch des Osteosynthesematerials am Unterkieferkorpus eine Revision und Reosteosynthese mittels 2 Miniplatten von enoral durchgeführt ( $\triangleright$ Abb. $\mathbf{4 b}$ b) c). Im weiteren Verlauf entwickelte sich eine infizierte Pseudoarthrose, sodass etwa 1 Jahr später eine erneute Revision im Sinne einer modellierenden Osteotomie, Augmenta-

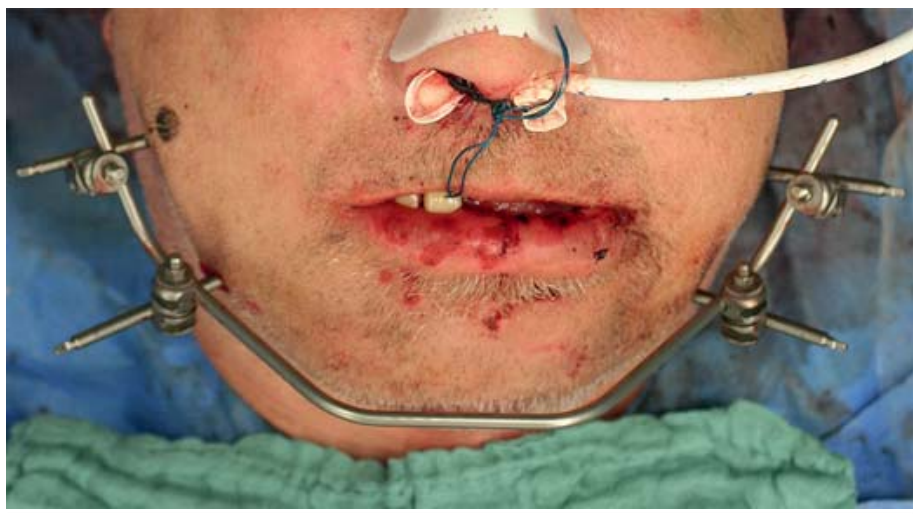

- Abb. 2 Fixateur externe zur primären Stabilisierung einer Unterkiefertrümmerfraktur in situ.
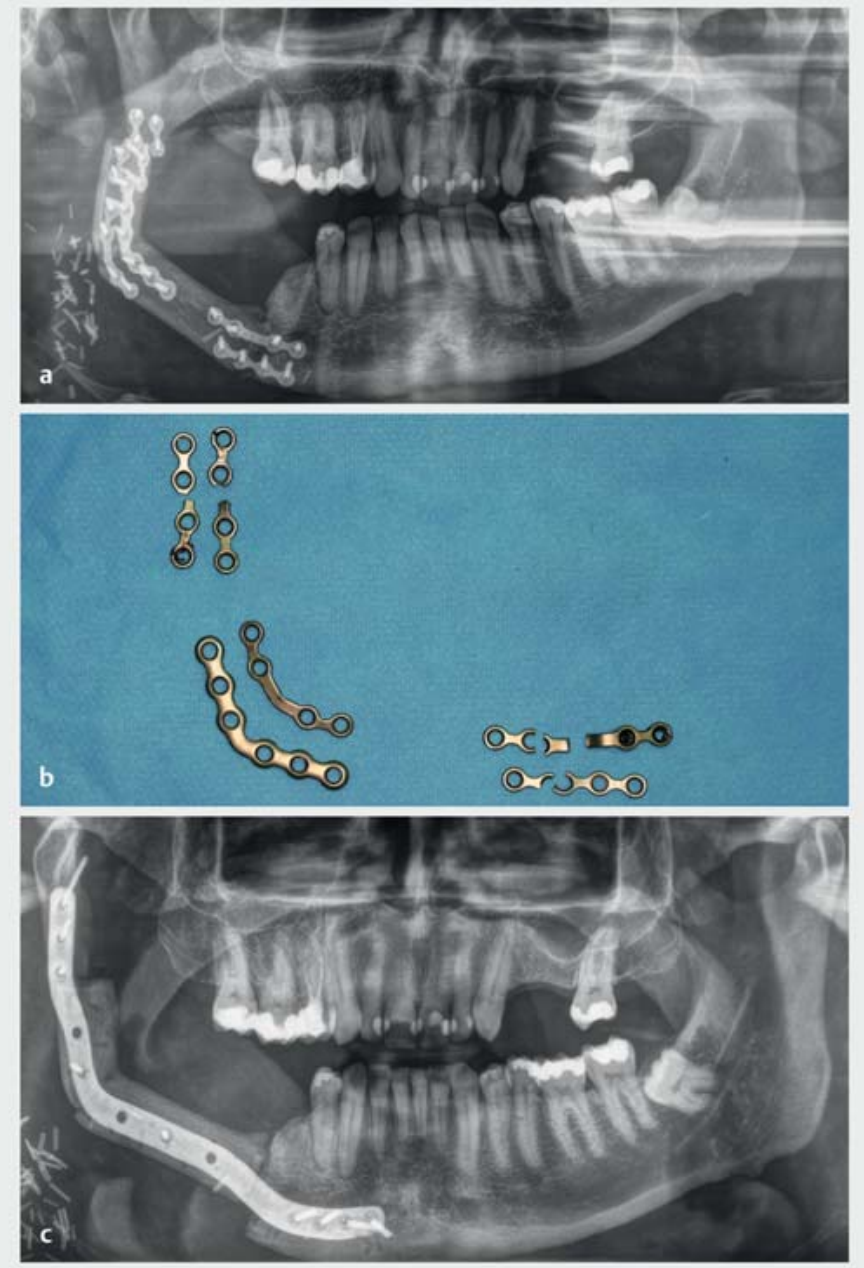

- Abb. 3 a Orthopantomogramm nach Unterkieferrekonstruktion mit einem mikrovaskulär reanastomosierten Fibulatransplantat und Fixation über Miniplatten mit Bruch des Osteosynthesematerials. Quelle: Charité, Radiologie Datenbank. b Explantiertes gebrochenes Plattenmaterial. c Orthopantomogramm nach Revision und Neuausrichtung des Fibulatransplantates und Rekonstruktion mittels eines patientenspezifischen Implantates (PSI). Quelle: Charité, Radiologie Datenbank. 

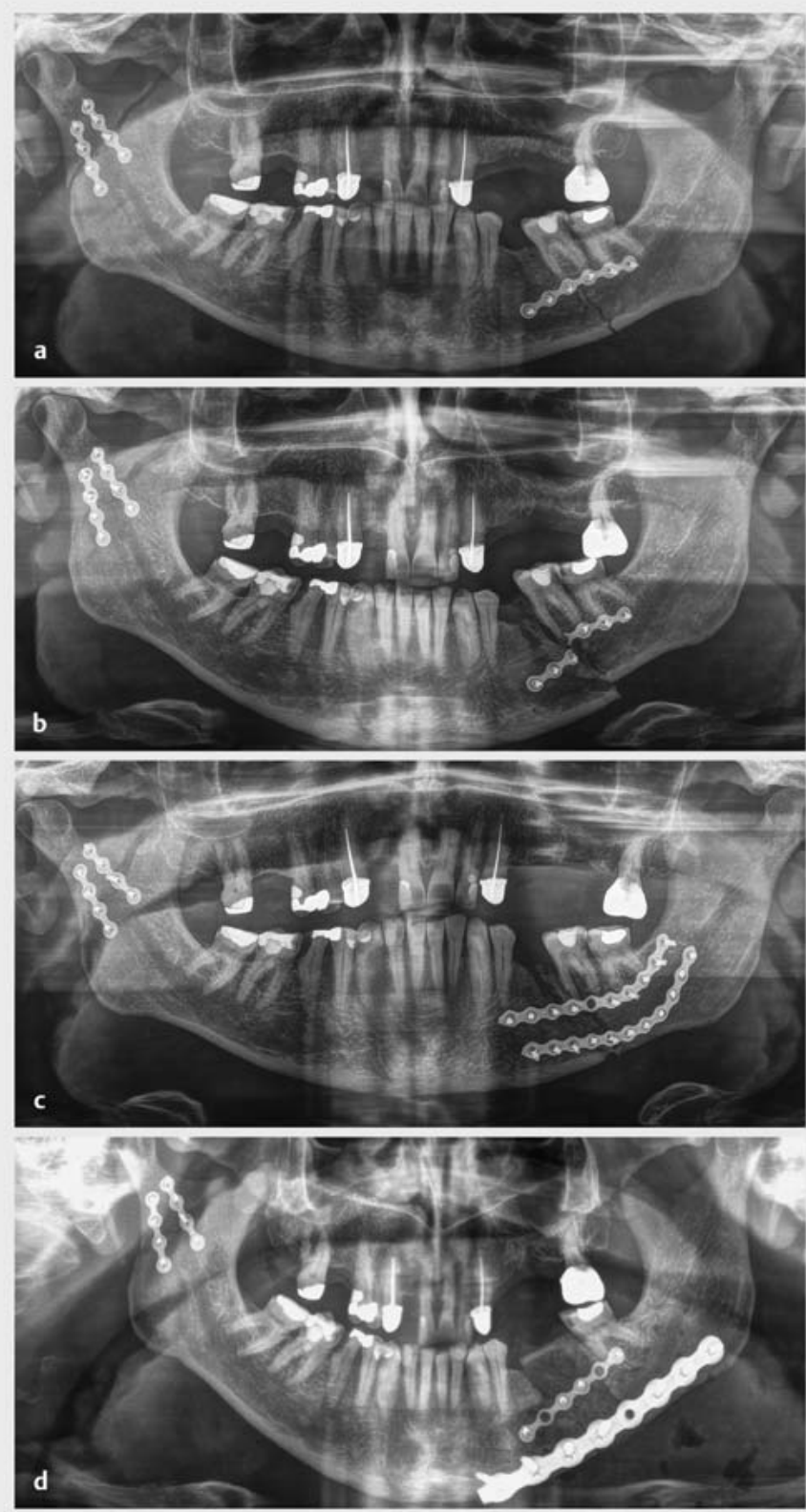

- Abb. 4 a Orthopantomogramm nach initialer Frakturversorgung mit Miniplattenosteosynthesen im Bereich des Unterkieferkorpus links und im Bereich der Gelenkfortsatzbasis rechts. b Orthopantomogramm mit Bruch der Osteosyntheseplatte im Bereich des Unterkieferkorpus links. c Orthopantomogramm mit Reosteosynthese durch 2 Miniplatten im Bereich des Unterkieferkorpus links. d Orthopantomogramm nach Revision und Augmentation mit 2 autologen kortikospongiösen Beckenkammspänen und Re-Reosteosynthese mit einer 2,7-mm-Rekonstruktionsplatte und einer 2,0-mm-6-Loch-Platte krestal. Quelle: Charité, Radiologie Datenbank.

tion mit 2 autologen kortikospongiösen Beckenkammspänen und Re-Reosteosynthese mit einer 2,7-mm-Rekonstruktionsplatte und einer 2,0-mm-6-Loch-Platte krestal im Sinne einer Stellschraubenosteosynthese beider Beckenkammtransplantate durch lange Schrauben
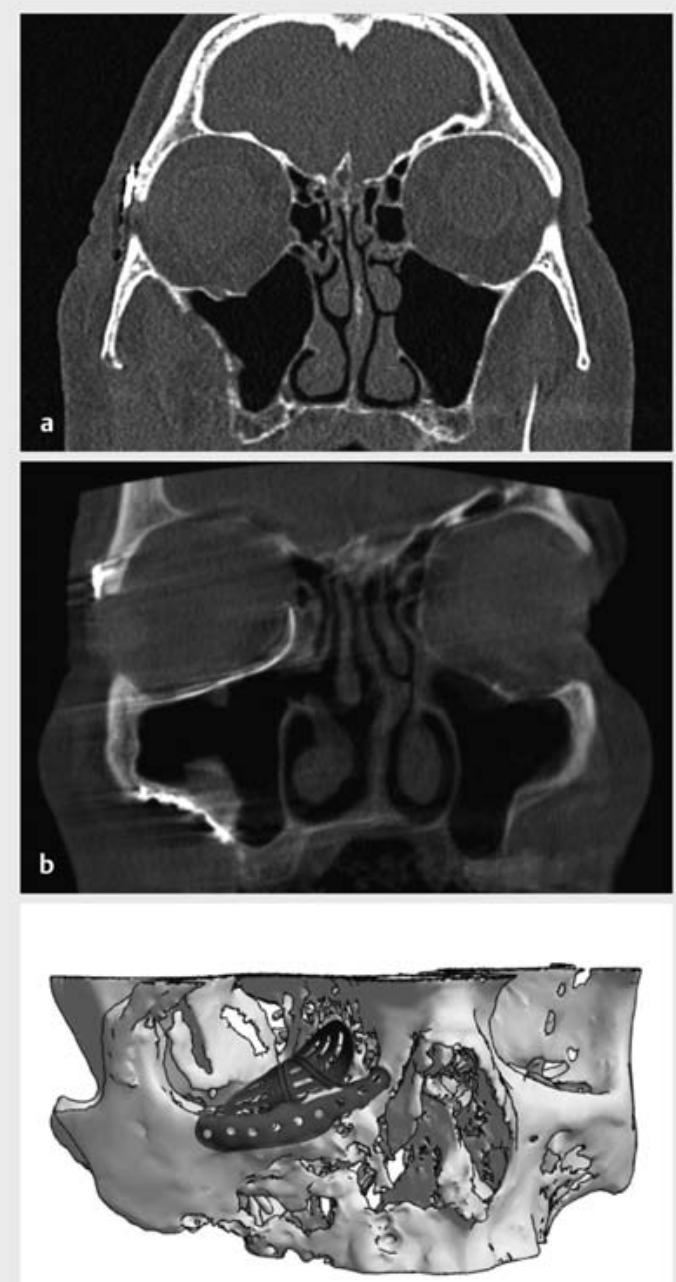

- Abb. 5 a Postoperative digitale Volumentomografie nach Rekonstruktion des Orbitabodens mittels EthisorbPatch. b Postoperative digitale Volumentomografie nach Revision des Defektes im Bereich des Orbitabodens mithilfe eines CAD-/CAM-gefertigten Titan-Meshes. c Computersimulation des CAD-/CAM-gefertigten patientenspezifischen Titanimplantates zur Rekonstruktion des Orbitabodens. Quelle: Charité, Radiologie Datenbank.

durchgeführt wurde ( $\triangleright$ Abb.4d). Im weiteren Verlauf zeigte sich die klinische Situation stabil. Alle weiteren Röntgenkontrolluntersuchungen zeigten ebenfalls einen stabilen Befund.

\section{Fall 3}

Im Rahmen der Versorgung einer lateralen Mittelgesichtsfraktur eines 85-jährigen Patienten mittels Miniplattenosteosynthese wurde eine Fraktur im Bereich des Orbitabodens initial mithilfe eines Ethisorb-Patches rekonstruiert ( $\boldsymbol{A}$ Abb. $\mathbf{5}$ a). Hierbei handelt es sich um ein resorbierbares Polyglactin/Poly-p-dioxanan, das bei kleineren Defekten regelhaft zur Rekonstruktion des nur pa- 


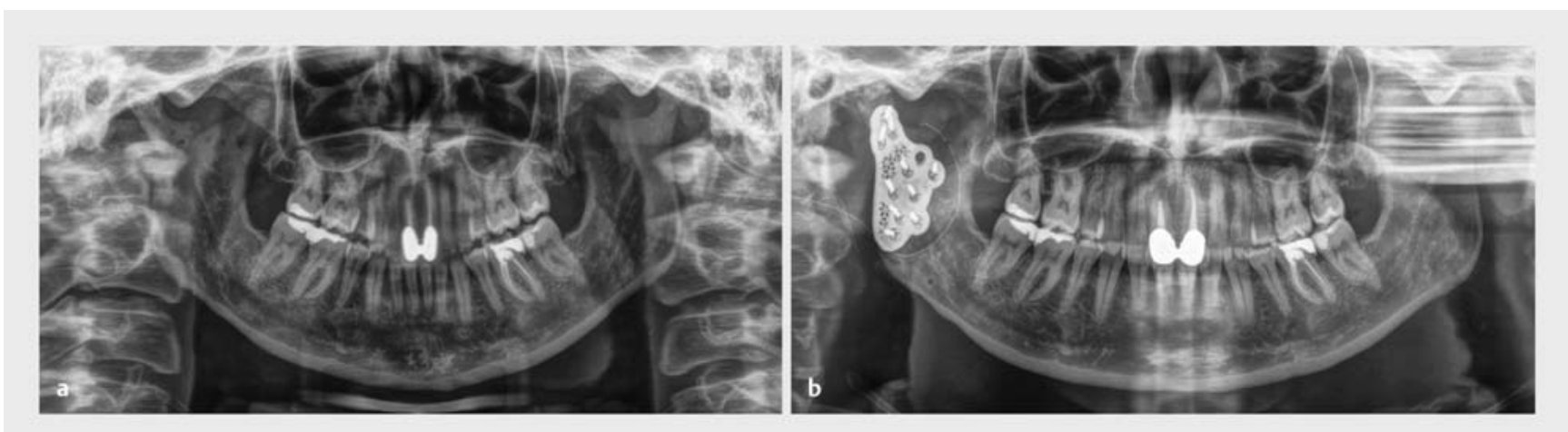

- Abb. 6 a Orthopantomogramm nach Entfernung des initial eingebrachten Osteosynthesematerials und Verheilung in Fehlstellung. b Orthopantomogramm nach Reosteotomie und Neuausrichtung des Gelenkfortsatzes sowie Einbringen des CAD-/CAM-gefertigten patientenspezifischen Implantates.

pierdünnen Bodens der Augenhöhle verwendet wird. Im weiteren Verlauf berichtete der Patient auch nach Abschwellen über persistierende Doppelbilder beim Aufblick. In der 3-D-Kontrolle mittels digitaler Volumentomografie zeigte sich ein erhöhtes intraorbitales Volumen ( $\bullet$ Abb. 5b). Wir entschieden uns zur Rekonstruktion des Orbitabodens durch ein CAD-/CAM-gefertigtes PSI aus Titan-Meshes ( $\mathbf{A b b} \mathbf{5} \mathbf{5} \mathbf{c}$ ). Als Vorlage diente die gespiegelte Orbita der Gegenseite. Postoperativ zeigte der Patient einen regelhaften ophthalmologischen Befund.

\section{Fall 4}

Die 40-jährige Patientin wurde in einem externen Krankenhaus im November 2017 bei einer Gelenkfortsatzfraktur nach synkopalem Sturz osteosynthetisch versorgt. Im Verlauf kam es zu einer Dislokation des Osteosynthesematerials, sodass eine Entfernung der Platten und Ruhigstellung mittels mandibulomaxillärer Fixation für 3 Wochen erfolgte. Im Anschluss berichtete die Patientin weiterhin über eine Störung der Okklusion, sodass wir uns etwa 6 Monate später zur Reosteotomie des Gelenkfortsatzes und Rekonstruktion mit einem CAD-/CAM-gefertigten patientenspezifischen Implantat entschieden ( $\triangleright$ Abb.6a,b). Der weitere postoperative Verlauf zeigte sich nach Implantation komplikationsfrei.

\section{Fazit}

Die Plattenosteosynthese aus Titan ist der Goldstandard in der Frakturversorgung der Mund-, Kiefer- und Gesichtschirurgie. Die Osteosyntheseplatten unterschiedlicher Stärke werden über intra- und extraorale Zugänge eingebracht. Trotz einer häufigen bakteriellen Besiedelung des Osteosynthesematerials spielen Infektionen eine untergeordnete Rolle. Ein schlechtes Knochenlager, zu gering dimensionierte Osteosyntheseplatten und eine Unterschätzung der biomechanischen Kräfte sind häufige Gründe für Komplikationen. In den meisten Fällen ist eine operative Revision erforderlich.
Autorinnen/Autoren
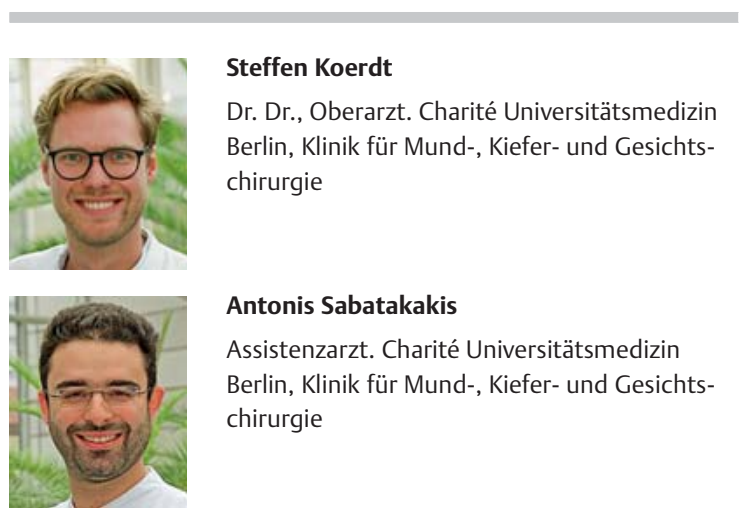

\section{Antonis Sabatakakis}

Assistenzarzt. Charité Universitätsmedizin Berlin, Klinik für Mund-, Kiefer- und Gesichtschirurgie
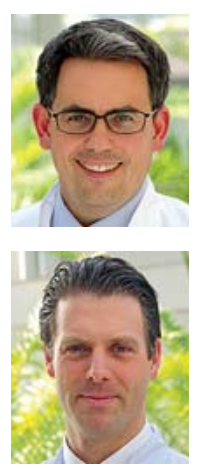

\section{Max Heiland}

Univ.-Prof. Dr. Dr., Klinikdirektor. Charité Universitätsmedizin Berlin, Klinik für Mund-, Kieferund Gesichtschirurgie

\section{Kilian Kreutzer}

Dr. Dr., Itd. Oberarzt. Charité Universitätsmedizin Berlin, Klinik für Mund-, Kiefer- und Gesichtschirurgie

\section{Korrespondenzadresse}

\section{Dr. med. Dr. med. dent. Steffen Koerdt}

Klinik für Mund-, Kiefer- und Gesichtschirurgie

Campus Benjamin Franklin

Charité Universitätsmedizin Berlin

Hindenburgdamm 30

12202 Berlin

Tel.: 030/84452501

Fax: 030/4507555929

steffen.koerdt@charite.de 


\section{Literatur}

[1] Motamedi MH, Dadgar E, Ebrahimi A et al. Pattern of maxillofacial fractures: a 5-year analysis of 8,818 patients. J Trauma Acute Care Surg 2014; 77: 630-634. doi:10.1097/ TA.0000000000000369

[2] Berenyi B. Aus der Geschichte der Behandlung der Gesichtsschädelfrakturen. In: Reichenbach E, Hrsg. Traumatologie im Kiefer-Gesichts-Bereich. Leipzig: Johann Ambrosius Barth; 1969

[3] Augat P, von Rüden C. Evolution of fracture treatment with bone plates. Injury 2018; 49 (Suppl. 1): S2-S7. doi:10.1016/ S0020-1383(18)30294-8

[4] Meara DJ, Jones LC. Controversies in maxillofacial trauma. Oral Maxillofac Surg Clin North Am 2017; 29: 391-399. doi:10.1016/j.coms.2017.06.002

[5] Champy M, Lodde JP, Schmitt R et al. Mandibular osteosynthesis by miniature screwed plates via a buccal approach. J Maxillofac Surg 1978; 6: 14-21

[6] Langford RJ, Frame JW. Tissue changes adjacent to titanium plates in patients. J Craniomaxillofac Surg 2002; 30: 103107. doi: $10.1054 / j c m s .2002 .0286$

[7] Langford RJ, Frame JW. Surface analysis of titanium maxillofacial plates and screws retrieved from patients. Int J Oral Maxillofac Surg 2002; 31: 511-518. doi:10.1054/ijom.2002.0283
[8] Piozzi R, Ribeiro DA, Padovan LE et al. Genotoxicity and cytotoxicity in multiple organs induced by titanium miniplates in Wistar rats. J Biomed Mater Res A 2009; 88: 342-347. doi:10.1002/jbm.a.31876

[9] Yerit KC, Hainich S, Enislidis G et al. Biodegradable fixation of mandibular fractures in children: stability and early results. Oral Surg Oral Med Oral Pathol Oral Radiol Endod 2005; 100: 17-24. doi:10.1016/j.tripleo.2004.11.013

[10] Champy M, Lodde JP, Grasset D et al. Ostéosynthèses mandibulaires et compression. Ann Chir Plast 1977; 22: 165-167

[11] Peled M, Ardekian L, Abu-el-Naaj I et al. Complications of miniplate osteosynthesis in the treatment of mandibular fractures. J Craniomaxillofac Trauma 1997; 3: 14-17

[12] Nakamura S, Takenoshita Y, Oka M. Complications of miniplate osteosynthesis for mandibular fractures. J Oral Maxillofac Surg 1994; 52: 233-238; discussion 238-239. doi:10.1016/0278-2391(94)90289-5

[13] Zachariades N, Papademetriou I, Rallis G. Complications associated with rigid internal fixation of facial bone fractures. J Oral Maxillofac Surg 1993; 51: 275-278. doi:10.1016/s0278-2391 (10)80174-0

\section{Bibliografie}

DOI https://doi.org/10.1055/a-1024-5927 online publiziert 17.12.2019 | OP-JOURNAL 2020; 36: 153158 ๔ Georg Thieme Verlag KG Stuttgart · New York ISSN 0178-1715 